\title{
Geriatric Assessment for the Nephrologist
}

\author{
Jocelyn Wiggins* and Markus Bitzert \\ "Department of Internal Medicine, Division of Geriatrics, University of Michigan Health System, Ann Arbor, \\ Michigan, 'Department of Internal Medicine, Division of Nephrology, University of Michigan Health System, \\ Ann Arbor, Michigan
}

\begin{abstract}
Dialysis providers are increasingly being presented with progressively older and frailer patients, in all healthcare settings from the acute hospital to the community dialysis center. These patients commonly bring more than kidney failure with them, with a complex constellation of chronic illness, comorbidity, and functional and cognitive impairment. Navigating these
\end{abstract}

challenges and coordinating the care of these highly complex patients significantly increase the work of the whole dialysis team. This article reviews the role of Comprehensive Geriatric Assessment in these patients and discusses how each of its elements interacts with routine dialysis care.
The size of the over 65-year-old population in the United States is growing rapidly and it is expected to double within the next 20 years. US Census Bureau data record an average life expectancy in 2004 of 75.2 years for men and 80.4 years for women; by 2015 , it is expected to be 76.2 years and 82.2 years, respectively, and to continue growing. This older age group is the largest consumer of healthcare services. Data collected by USRDS (US Renal Data System) demonstrate that End-Stage Kidney Disease (ESKD) is a disease of the older population, with numbers starting to rise significantly after the age of 50 (1). Mean age at the start of renal replacement therapy is 62.3 years for men and 63.4 years for women. Peak incident counts of treated ESKD occur in the 70-79 age group at over 15,000 patients per year. Incident rate of treated ESKD in the 70-79 age group is 1,673 per million person-years and peaks at $80-85$ years of age at 1,931 per million population. More than 55\% of ESKD patients are more than 60 years old. These statistics demonstrate the need for every nephrologist to feel comfortable in the role of a geriatrician and know how to meet the special needs of their older patients.

Older patients typically bring to the dialysis unit a complex burden of comorbidity and problems that complicate the diagnosis and management of their kidney disease. When considering the older individual, it is important to be able to determine what findings constitute normal aging versus disease. Furthermore, older adults are statistically more likely to have several common diseases, rather than a single rare disease explaining their symptoms and therefore the principles of Occam's

Address correspondence to: Jocelyn Wiggins, Department of Internal Medicine, Division of Geriatrics, University of Michigan, Ann Arbor, Ml 48109, Tel.: 734-615-4460, Fax: 734763-0982, or e-mail: wiggi@umich.edu.

Seminars in Dialysis-Vol 25, No 6 (November-December) 2012 pp. 623-627

DOI: $10.1111 /$ sdi. 12027

(C) 2012 Wiley Periodicals, Inc.
Razor (diagnostic parsimony) do not necessarily apply. The Comprehensive Geriatric Assessment was first developed in the 1960 s to address this problem, and many studies were published in the 1970s as this concept was developed and refined. More recent studies include those by Harvey Cohen in the US (2) and Graham Ellis in Europe (3). The primary goal of comprehensive geriatric assessment is to help the patient regain lost function and maintain as much independence as possible. It is important to note that physical and mental illnesses affecting the elderly often interact and result in a loss of functional ability that is much greater than any one problem in itself. The elderly are particularly vulnerable as they have less "reserve capacity".

\section{Goals of Comprehensive Geriatric Assessment}

1. To provide a long-term plan for patients who have multiple physician and ER visits and hospital admissions

2. To focus on preventative strategies

3. Improving/maintaining functional ability and not necessarily a "cure"

4. To establish plans for coordination of care

5. To determine the need for and site of a structured living environment

6. To determine optimal use of healthcare resources

7. To prevent re-admission to the hospital

The team performing the assessment may include representatives from medicine, nursing, dentistry, dietary, social work, audiology/speech, physical and occupational medicine, psychology/psychiatry, among others. This will be familiar territory to the nephrologist, who is accustomed to working with a multidisciplinary team. Patients receiving hemodialysis are in the dialysis unit three times per week. Therefore, there are plenty of 
opportunities to perform some of the tests during these visits. "Geriatric rounds" in a dialysis unit may be an efficient way to manage geriatric aspects of older adults receiving hemodialysis. Care plans developed after the Geriatric Assessment must be carefully coordinated to avoid conflicting plans, messages, or treatments. Proper and thorough communication is key to a successful outcome. It is enormously important to understand that no one individual is responsible for obtaining all the individual elements. The physician's role is to make sure that a full assessment is done and coordinate the implementation of the plan that is developed. Many of the pieces of the plan will be handed off to other care providers with the appropriate expertise.

\section{Components of a Comprehensive Geriatric Assessment}

1. Diagnoses and Problems: assessment to include oral health, vision, hearing, and gait/posture, falls

2. Detailed review of all prescription and over-thecounter medications including supplements/herbs

3. Nutritional Assessment-including weight and nutritional indices

4. Assessment of Bowel Function

5. Assessment of Cognitive Ability and capacity for informed decision making

6. Mental/Emotional Health

7. Functional abilities: ADLs and IADLs

8. Assessment regarding ability to adhere to Prescribed Care Plan

9. Spiritual Needs/Beliefs

10. Existing Community Support Structure

11. Current and anticipated Living Environment

12. Legal Issues_-Living Wills, Durable Power of Attorney, End-of-Life Issues

Below we will discuss some of the issues that are most relevant to managing geriatric patients with ESKD on dialysis.

\section{Functional Assessment}

Improving or maintaining the functional status of older adults is one of the main goals in Geriatric Medicine. The first step toward that goal is an accurate assessment of the functional status of each patient to determine the ability to perform in daily life and identify specific deficits that may require intervention. The overall functional status of an older adult is estimated by determining their ability to perform routine tasks of daily life or Activities of Daily Living (ADL). ADLs are further subdivided into Basic ADLs and Instrumental ADLs referred to as IADLs. Basic ADLs include mobility, bathing, dressing, toileting, transferring, continence, and feeding.

Loss of ADL ability is a bad prognostic sign as it implies significant debility and dependence. According to US Census Bureau data, $40 \%$ of the over 65 -year olds have some level of disability, and this will have an impact on their medical care. IADLs include the ability to use the telephone, shop, prepare food, housekeeping, laundry, transportation, taking medication, and managing money. These skills are vital to compliance of the renal patient as they are necessary for the patient to be able to access dialysis and follow a complex medical regimen and dietary restrictions.

Many frail and older patients undergo a progressive decline in function after starting dialysis, and unit staff may express concern about their ability to care for themselves and whether they are in a sufficiently supportive environment. These patients can be referred to occupational therapy for a Kohlman Evaluation of Living Skills (KELS) evaluation. KELS assesses ADLs in the following five areas: self-care, safety and health, money management, transportation and telephone, and work and leisure (4). The results of this testing can then be used to determine whether the patient is capable of caring for themselves and the patient and family can be counseled accordingly. This is especially useful with patients who have difficulty with keeping appointments, taking their medications correctly, and following dietary restrictions.

\section{Cognitive Assessment}

Intact cognitive function is critical to perform the complex tasks expected from dialysis patients. As there is a well-established link between aging and dementia, the major concern is to screen for dementia as patients age. Compared with the general population, the rates of cognitive impairment are three to four times higher among patients with ESKD. The incidence of cognitive impairment starts to manifest when the eGFR falls below $45 \mathrm{ml} /$ minute $(5,6) ; 70 \%$ of dialysis patients over 55 have some level of cognitive impairment with $10-20 \%$ suffering from frank dementia. In the general population, more than $70 \%$ of incident dementia is of the Alzheimer's type, whereas patients with CKD and ESKD are more likely to develop vascular dementia.

Screening for dementia in the general population is usually performed using the Folstein Mini-Mental State Exam (7). The major concern in the dialysis population is to identify patients with executive dysfunction not able to manage complex medical regimen and not competent to sign their own consent forms. For more specific evaluation for executive dysfunction, the more recently developed Montreal Cognitive Assessment (MoCA) is recommended (8), as it is a much more sensitive tool for patients with kidney disease. Furthermore, it can be administered by any member of the care team. Although pharmacologic intervention has a limited role in ESKD patients with cognitive impairment, a low score should prompt concerns about the patient's ability to make his/her own medical decisions and his/her ability to follow the recommended regimen, and thus may impact planning for future care needs. Cognitive impairment and kidney disease share many risk factors, but albuminuria, anemia, intradialytic hypotension, and dialysis disequilibrium contribute to cognitive decline (9). The role of targeting ESKD-associated risk factors to 
improve cognitive function or at least halt cognitive decline is not yet established.

\section{Polypharmacy}

If following evidence-based guidelines, most renal patients will inevitably meet criteria for polypharmacy. The contributing factors include increased burden of comorbidities often managed by several physicians and care teams using multiple medications, and altered pharmacokinetics due to age-associated changes in metabolism and body composition, and by hemodialysis treatments. Thus, the possibility for adverse drug interactions and reactions is strongly increased.

Keeping track of which medications patients are actually taking including supplements, vitamins, and nutraceuticals is a daunting problem. The standard recommendation is that patients bring all of their medicines to clinic or dialysis appointments. This can be unrealistic and a better strategy is to have a nurse or pharmacy tech call the patient at home, where they can sit down with the contents of the medicine cabinet and do a thorough audit.

The Beers criteria for inappropriate prescribing in the elderly, originally published in 1991, have been revised several times since then, and most recently by the American Geriatric Society in $2012(10,11)$. It serves as a useful reference when patients and families are concerned about side effects or drug interactions. Patient needs and their circumstances should be kept in mind when using the criteria, as it is sometimes necessary to use less desirable medications for optimal medical management.

\section{Mental and Emotional Health}

Depression is common in the elderly population and compounded by the prevalence of chronic disease burden. The true incidence of depression in the older dialysis population is unclear. Different studies suggest different prevalence rates. This is partly because different instruments for identifying depression are used and each instrument detects a different segment of the population of patients with depression, and therefore, results depend on the test used. Some studies have shown rates as high as $45 \%$. In addition, the identification and assessment of symptoms of depression are also complicated by the overlap with symptoms of uremia and cognitive decline. Many of the somatic manifestations of depression, such as fatigue, loss of energy and motivation, and poor appetite are similar to those of underdialysis, uremia, or progressively impaired cognition. Furthermore, coping with the fact of requiring dialysis, and the manifold requirements and restraints including spending significant proportions of the awake time in a hemodialysis center or for health-related tasks, (peritoneal dialysis) can promote depressive symptoms.

Diagnosis and treatment of depression are important for many reasons. There are clear links between poor compliance and depression. Several studies have now shown a link between the presence of depression and dialysis mortality. Depression also is a significant contributor to poor quality of life in dialysis patients $(12-14)$. Tests to detect depression can be administered by any team member and should be performed on a regular basis on all dialysis patients. Developing strategies to improve symptoms in ESKD patients is complex, as the etiology of depression is likely multi-factorial, and contributing issues have to be addressed as part of a comprehensive care plan.

\section{Mobility, Gait, and Falls}

Mobility is important when patients have a need to leave the home to access the dialysis facility and sit in a chair for several hours. Mobility is complex and affected by many different physical processes. As patients become more frail and lose muscle mass through deconditioning and dietary restriction, mobility becomes challenged. Add to this the many medications with potential side effects and dialysis itself with its shifts in volume, blood pressure, and electrolyte levels, and the patients' ability to maintain normal mobility and gait is likely to be compromised. There are a limited number of studies about these issues and their consequences.

The most serious complication of decreased mobility is injurious falls. About one-third of communitydwelling seniors over 65 have falls each year and fully one half of seniors over 80 have falls. One in five of these falls results in significant injury. It is clear that falls occur at a higher rate in the dialysis population and that fractures are 4 times more common in ESKD population than in the general population. The risk for fractures is multiplied by the high prevalence of renal osteodystrophy in patients with ESKD. Furthermore, 1-year mortality in dialysis patients who have a hip fracture is 2-3 times that of an older community-dwelling person who has a similar fracture. A single fall in a dialysis patient over 65 years increases the risk of death after adjustment for comorbidities (15-17). These numbers underscore the urgency to improve or maintain mobility in older adults on dialysis, and again individualized interventions are most likely to be successful.

\section{Nutrition}

Nutritional assessment is a critical parameter routinely and closely monitored by Geriatricians and Nephrologists. Nephrologists are already very familiar with challenges that malnutrition presents in the dialysis unit, and have a dietician as part of their care team. Even though large-scale studies are limited, overnutrition, undernutrition, and malnutrition are very common in older adults on dialysis independent of modality and are associated with increased mortality.

Older adults have many risk factors for quantitative and qualitative nutritional deficits as aging, loss of kidney function. and dialysis independently impact 
metabolism and nutritional status. Furthermore, other functions that decrease with age like mobility and cognitive function can interfere with maintaining good nutritional status. Both extremes of body weight are represented in the elderly dialysis population. Almost $50 \%$ of the dialysis population has type 2 diabetes, many of them with related obesity. At the other end of the spectrum are the "little old ladies who survive on tea and toast". In this context, findings that patients initiating dialysis with somewhat higher BMI have a better outcome (reverse epidemiology) make BMI difficult to utilize for optimizing patient care. Independent of body weight, malnutrition can be due to imbalance of nutrients and may not be linked directly with BMI. Therefore, other parameters including serum albumin, physical endurance, overall functional status, and ability to enjoy food should be taken into consideration, and have to be addressed individually.

One special challenge in the older population that has to be addressed is access to nutritious foods. Many older patients are no longer able to access groceries for themselves and may rely on a friend or family member to shop for them. Some of them may be unable, or unwilling to cook for themselves and rely on frozen or prepared foods. Some may live in congregate housing environments where meals are provided, and special diets cannot be accommodated. Cognitive impairment can impact a patient's ability to follow dietary restrictions. For all of these reasons, nutrition is a greater challenge in the older than in the younger dialysis population and should be kept in mind when managing these patients. Giving patients and their families realistic goals for dietary management that acknowledge the barriers should be part of the care plan.

\section{Sensory Screening}

Both vision and hearing deteriorate with age and are important for successful communication with the patient, for completing complex tasks required to maintain his health, including taking medications, and to maintain mobility and cognitive function. About $40 \%$ of the community-dwelling over 65 -year-old population has visual deficits. Common visual problems include glaucoma, age-related macular degeneration, cataracts, and diabetic retinopathy (18). Visual loss impacts function, cognition, mobility and quality of life. Dialysis care should include making sure that patients have regular ophthalmologic screening. Early treatment of all of these problems can control visual loss and help maintain patient function.

Similarly about one-third of older patients have hearing impairment (19). This also has a major impact on patient cognition and on emotional, physical, and social function. Amplification can be helpful and the patient should be sent for audiology screening if there are concerns. Unlike visual aids, Medicare does not pay for hearing aids and the cost of the newer digital aids is very high.

\section{Advance Care Planning}

Discussion around end-of-life planning should happen early in dialysis care and be repeated whenever there is a change in medical or functional status. It is important to make sure that all team members are aware of patient's goals and preferences should they become unable to speak for themselves. These discussions should include identification of the surrogate decision maker in addition to the patient's preferences around end-of-life care and resuscitation. These issues will be dealt with in detail in another article, but every dialysis provider should take responsibility for ensuring that they are carried out and the results recorded and shared with the entire care team.

\section{Conclusion}

Comprehensive geriatric assessment should happen at the time a patient initiates dialysis treatment, and results should be discussed with the entire team. All team members should feel comfortable contributing to the resulting plan. It can be done sequentially over many visits; there is no reason to complete the entire process in one sitting. It should be repeated whenever there is a major change in a patient's health or functional status. Hospitalizations, emergency room visits, and major events in the unit should trigger the team to reassess all or parts of the assessment and modify the plan based on the results of the re-evaluation.

\section{Acknowledgment}

Markus Bitzer is supported by R03 AG040651 from the National Institute of Aging of the National Institutes of Health.

\section{References}

1. U.S. Renal Data System, USRDS: 2008 Annual Data Report: Atlas of End-Stage Renal Disease in the United States. Bethesda, MD: National Institutes of Health, National Institute of Diabetes and Digestive and Kidney Diseases, 2008

2. Cohen HJ, Feussner JR, Weinberger M, Carnes M, Hamdy RC, Hsieh F, Phibbs C, Courtney D, Lyles KW, May C, McMurtry C, Pennypacker L, Smith DM, Ainslie N, Hornick T, Brodkin K, Lavori P: A controlled trial of inpatient and outpatient geriatric evaluation and management. N Engl J Med 346(12):905-912, 2002

3. Ellis G, Whitehead MA, Robinson D, O'Neill D, Langhorne P: Comprehensive geriatric assessment for older adults admitted to hospital: meta-analysis of randomised controlled trials. BMJ 343:d6553, 2011. doi: $10.1136 /$ bmj.d6553

4. Kohlman-Thomson L: Kohlman Evaluation of Living Skills. Bethesda, MD: American Occupational Therapy Association, 1992

5. Murray AM: Cognitive impairment in the aging dialysis and chronic kidney disease populations: an occult burden. Adv Chronic Kidney Dis 15(2):123-132, 2008. Review

6. Madero M, Sarnak MJ: Does hemodialysis hurt the brain? Semin Dial 24(3):266-268, 2011

7. Folstein MF, Folstein SE, McHugh PR: "Mini-mental state". A practical method for grading the cognitive state of patients for the clinician. J Psychiatr Res 12(3):189-198, 1975

8. Nasreddine ZS, Phillips NA, Bédirian V, Charbonneau S, Whitehead V, Collin I, Cummings JL, Chertkow H: The Montreal Cognitive Assessment, MoCA: a brief screening tool for mild cognitive impairment. J Am Geriatr Soc 53(4):695-699, 2005 
9. Kurella Tamura M, Yaffe K: Dementia and cognitive impairment in ESRD: diagnostic and therapeutic strategies. Kidney Int 79(1):14-22, 2011. Epub 2010 Sep 22. Review

10. Beers MH, Ouslander JG, Rollingher I, Reuben DB, Brooks J, Beck JC: Explicit criteria for determining inappropriate medication use in nursing home residents. UCLA Division of Geriatric Medicine. Arch Intern Med 151(9):1825-1832, 1991

11. The American Geriatrics Society: Beers Criteria Update Expert Panel. American Geriatrics Society updated beers criteria for potentially inappropriate medication use in older adults. $J$ Am Geriatr Soc 60(4):616-631, 2012

12. Halen NV, Cukor D, Constantiner M, Kimmel PL: Depression and mortality in end-stage renal disease. Curr Psychiatry Rep 14(1):36-44, 2012

13. Fischer MJ, Kimmel PL, Greene T, Gassman JJ, Wang X, Brook DH, Charleston J, Dowie D, Thornley-Brown D, Cooper LA, Bruce MA, Kusek JW, Norris KC, Lash JP: AASK Study Group. Elevated depressive affect is associated with adverse cardiovascular outcomes among African Americans with chronic kidney disease. Kidney Int 80(6):670-678, 2011
14. Cukor D, Cohen SD, Peterson RA, Kimmel PL: Psychosocial aspects of chronic disease: ESRD as a paradigmatic illness. J Am Soc Nephrol 18(12):3042-3055, 2007

15. Desmet C, Beguin C, Swine C, Jadoul M: Falls in hemodialysis patients: Prospective study of incidence, risk factors, and complications. Am J Kidney Dis 45:148-153, 2005

16. Cook WL, Tomlinson G, Donaldson M, Markowitz SN, Naglie G, Sobolev B, Jassal SV: Falls and fall-related injuries in older dialysis patients. Clin J Am Soc Nephrol 1:1197-1204, 2006

17. Jadoul M, Albert JM, Akiba T, Akizawa T, Arab L, Bragg-Gresham JL, Mason N, Prutz KG, Young EW, Pisoni RL: Incidence and risk factors for hip or other bone fractures among hemodialysis patients in the Dialysis Outcomes and Practice Patterns Study. Kidney Int 70:13581366,2006

18. Eichenbaum JW: Geriatric vision loss due to cataracts, macular degeneration, and glaucoma. Mt Sinai J Med 79(2):276-294, 2012

19. Lin FR: Hearing loss in older adults: who's listening? JAMA 307(11):1147-1148, 2012 